\title{
Ian Kershaw
}

\section{Adolf Hitler und die Realisierung der nationalsozialistischen Rassenutopie}

Streng genommen wurde unter dem Nationalsozialismus keine Utopie realisiert, außer wenn man darunter Massenmord - die Ermordung von beinahe sechs Millionen Juden und zahllosen anderen "Untermenschen" - versteht. Und streng genommen war das Programm der Nationalsozialisten auch keine Utopie - jedenfalls nicht im Sinne von Thomas Morus. In seinem berühmten Werk aus dem Jahre 1516 umriß der englische Staatsmann, Jurist und Denker ganz konkrete Vorstellungen von einer imaginären Gesellschaft, die er "Nirgendwo" (Utopia) nannte. Das Wesentliche daran war die Sicherstellung von Gerechtigkeit für alle Mitbürger, garantiert durch ein staatliches System, das auf Rationalität, Frieden, Recht, Ordnung und Gleichheit - Privateigentum war nicht vorgesehen - beruhte. Die Gestalt dieses politischen und gesellschaftlichen Systems wurde von Morus klar herausgearbeitet und dargestellt.

Wie wenig davon hat einen direkten Bezug auf Hitler! Der deutsche Diktator unterhöhlte jede Basis eines Rechtsstaats und alle grundsätzlichen Strukturen eines staatlichen Gebildes. Ein administratives Chaos war die Folge. Noch wichtiger: Frieden, Gleichheit, Gerechtigkeit sind Begriffe, die man schwerlich mit Hitler assoziieren kann. Die materielle Korruption, die nach Morus ein Grundübel jeder Gesellschaft bildet und auf das Privateigentum zurückzuführen ist, blühte im Dritten Reich wie nie zuvor. Hitler hatte also, kurz gesagt, keine Utopievorstellung im klassischen Sinne des Wortes.

Im übertragenen Sinne kann man im Zusammenhang mit Hitler aber wohl doch von utopischem Denken sprechen - wenn man sich darunter eher vage, visionäre Fernziele vorstellt. Der Mangel an Konkretheit dieser Fernziele war für Hitler sogar äußerst vorteilhaft. Er hatte zur Folge, daß die Fernziele unterschiedliche Bedeutung für verschiedene Leute zu verschiedenen Zeiten erlangen konnten. Die utopischen Fernziele galten deswegen in der Regel als Integrationsfaktoren und als Aktionsrichtungen - eine für einen wachsenden Teil der deutschen Gesellschaft höchst attraktive Zukunftsvision, eine Zielvorstellung, die gerade durch ihre Vagheit breite Anziehungskraft hatte und große Energien entfesseln konnte; konkreten Inhalt gewann diese Zielvorstellung dagegen erst im Lauf der Zeit.

Die einzelnen Bestandteile dieser Vision bedürfen hier wohl keiner ausführlichen Erläuterung. Äußerst unbestimmt war die Vorstellung von einer besseren 
Gesellschaft, einer vereinten, starken, wohlhabenden, harmonischen „Volksgemeinschaft": Beim Aufstieg der NSDAP Anfang der 30er Jahre war dies die positivste und attraktivste utopische Idee im Angebot der NS-Propaganda. Auch nach der Machtübernahme blieb die affektive Anziehungskraft der Volksgemeinschaftsidee aufrechterhalten. Aber an konkretem, konstruktivem Inhalt jenseits vom ${ }_{n}$ schönen Schein des Dritten Reiches ${ }^{\prime}$, jenseits der suggestiven Propaganda, jenseits von Ritualen, Veranstaltungen, Symbolen usw. war kaum etwas von einer echten Gemeinschaft zu spüren. Und für Hitler selbst blieb die Idee erst recht inhaltsleer - und nützlich hauptsächlich in ihrer mobilisierenden Funktion. Klar umrissen war die Idee aber doch, freilich in einem negativen Sinne - in der Ausschaltung von "schädlichen Elementen", insbesondere in der "Säuberung " von "fremdem Blut ". Denn die Volksgemeinschaft nahm nur in ihrer Exklusivität Gestalt an, in der Ausgrenzung von denjenigen, die nicht dazugehörten. Und an diesem Punkt kommen wir zum Kern von Hitlers Utopievorstellungen.

Schon im September 1919 sah Hitler „unverrückbar die Entfernung der Juden überhaupt " als Hauptziel einer nationalen Regierung an ${ }^{2}$. Was genau das bedeutete, und wie das Ziel zu erreichen sei, erläuterte Hitler nicht. $Z u$ verstehen war darunter wohl, daß in langfristiger Perspektive die Juden aus der deutschen Gesellschaft ausgegrenzt und schließlich vertrieben werden sollten. So jedenfalls verstand auch die NS-Führung die Judenpolitik bis zum Kriegsausbruch. Viele Instanzen und Behörden auf verschiedenen Ebenen versuchten, dieses Fernziel in die Realität umzusetzen, jedoch ohne daß das Ziel näher und präzise definiert wurde. Und erst allmählich, im Zeichen der Entfaltung seiner expansionistischen Ziele in der zweiten Hälfte der 30 er Jahre, begann Hitler selbst sich bedrohlich zu äußern - und dann auch nur andeutungsweise, z. B. gegenüber Joseph Goebbels: „Die Juden müssen aus Deutschland, ja aus ganz Europa heraus. Das dauert noch eine Zeit, aber geschehen wird und muß das. ${ }^{3}{ }^{3}$ Konkrete Pläne für ein umfassendes Programm zur physischen Vernichtung der europäischen Juden entwickelten sich, wie wir wissen, erst im Jahre 1941 - was Hans Mommsen als „die Realisierung des Utopischen" bezeichnete ${ }^{4}$.

Für Hitler war die "Entfernung der Juden" kein in sich geschlossenes und eigenständiges Ziel. Eher war sie der zentrale Bestandteil von einer breiteren utopischen Konzeption, die zunächst den Wiederaufbau Deutschlands beinhaltete, dann die restlose und endgültige Zerschlagung seiner Feinde, die Sicherung der

1 Peter Reichel, Der schöne Schein des Dritten Reiches. Faszination und Gewalt des Faschismus (Frankfurt a.M. 1993).

2 Eberhard Jäckel, Axel Kubn (Hrsg.), Hitler. Sämtliche Aufzeichnungen 1905-1924 (Stuttgart 1980) 88-90.

${ }_{3}$ Tagebucheintrag von Goebbels vom 30. 11. 1937, in: Elke Fröblich (Hrsg.), Die Tagebücher von Joseph Goebbels. Sämtliche Fragmente. Teil 1, Bd. 3, Aufzeichnungen 1924-1941 (München 1987$) 351$.

4 Hans Mommsen, Die Realisierung des Utopischen: Die „Endlösung der Judenfrage“ im „Dritten Reich“, in: GG 9 (1983) 381-420; im folgenden zitiert: Mommsen, Realisierung des Utopischen. 
materiellen Basis für die Zukunft durch Eroberung von "Lebensraum “im Osten und nach der Erlangung der deutschen Vorherrschaft in Europa schließlich die Vorherrschaft in der Welt. Deswegen war sich Hitler seit spätestens Mitte der 20er Jahre im klaren darüber, daß die Basis für die Realisierung dieser Utopie in absehbarer Zeit ein Krieg gegen den ,jüdischen Bolschewismus“, wie er das sowjetische System regelmäßig bezeichnete, sein mußte. Das bedeuter: Die Utopie war für Hitler im Endeffekt der Krieg und das Endziel unbeschränkte Macht über nicht näher definierte eroberte Gebiete. Der Erste Weltkrieg markierte für ihn, wie er oft zu sagen pflegte, den bisherigen Höhepunkt seines Lebens. Die kommende große Auseinandersetzung mit dem Erzfeind sollte die Verwirklichung seiner Utopie mit sich bringen. Die deutsche Gesellschaft mußte für diesen Krieg materiell und psychologisch gerüstet sein. Dies war im Grunde genommen das einzige Ziel der NS-Innenpolitik zwischen 1933 und 1939.

Da für Hitler nur die Stärke zählte, und da die Stärke in seinen Augen nur von der Qualität der Rasse abhing, verstand es sich von selbst, daß rassische Säuberung und Auslese die Grundlagen seiner utopischen Vorstellung bildeten: „Würde Deutschland jährlich eine Million Kinder bekommen und 700000-800000 der schwächsten beseitigen", erklärte Hitler auf dem Nürnberger Parteitag 1929, "dann würde am Ende das Ergebnis vielleicht sogar eine Kräftesteigerung sein ${ }^{45}$. Gefährlicher als alles andere für die Kraft und Stärke eines Volkes war nach seiner Sicht die Blutvermischung: „Alle großen Kulturen der Vergangenheit gingen nur zugrunde, weil die ursprünglich schöpferische Rasse an Blutvergiftung abstarb“, schrieb er in "Mein Kampf ${ }^{\text {"6 }}$. Aber er blieb auch hier in seinen rassistischen $\mathrm{Ge}$ danken recht vage. Nur die "Arier" und die Juden waren letztlich erwähnenswert in seiner Interpretation vom ewigen darwinistischen Rassenkampf, der nach seiner Meinung die Grundlage der menschlichen Existenz überhaupt ausmachte. Auch in den 30 er Jahren befaßte sich Hitler, anders als etwa Himmler und die sonstige SS-Führung, kaum mit pseudointellektuellen Fragen der Rassenpolitik. Alle nicht-arischen ethnischen Gruppen galten ihm selbstverständlich als "Untermenschen". Aber im Gegensatz zu seiner paranoiden Beschäftigung mit den Juden äußerte er sich z. B. recht wenig über die Tschechen, die Polen, die Slawen im allgemeinen oder die "Zigeuner" (Sinti und Roma). Auch hier war Hitlers Utopievorstellung nichts als rassistischer Imperialismus. Dic ferne Vergangenheit - die Zeit der Völkerwanderung - galt einerseits als Muster für seine Utopie: „Als Eroberer unterwarf er (der Arier, I.K.) sich die niederen Menschen und regelte dann deren praktische Betätigung unter seinem Befehl, nach seinem Wollen und für seine Ziele. "7 Noch bedeutender für die Gestaltung seiner Utopie war allerdings der Imperialismus des 19. Jahrhunderts. Das beste Beispiel für die künftige deutsche Utopie lieferte in Hitlers Augen die britische Herrschaft in Indien. „Der rus-

5 Adolf Hitler, Rede auf dem Nürnberger Parteitag (1929), in: ders., Reden, Schriften, Anordnungen, Bd. 3: Februar 1925 bis Januar 1933, hrsg. v. Institut für Zeitgeschichte (München 1992-98) 348.

6 Adolf Hitler, Mein Kampf (München ${ }^{171943)} 316$.

7 Ebd. 324. 
sische Raum ist unser Indien, und wie die Engländer es mit einer Handvoll Menschen beherrschen, so werden wir diesen unseren Kolonialraum regieren“, führte er in einem Monolog bei Tisch im September 1941 aus $^{8}$. Nun konnte er als Herrscher Europas seine fantastischen Utopievorstellungen voll entfalten: die Besiedlung der Ukraine und der Krim mit deutschen Wehrbauern; die restlose materielle Ausbeutung der Ostgebiete, um einen erhöhten Lebensstandard in Deutschland zu gewährleisten; den Ausbau von Autobahnen, um deutschen Touristen „die Schönheit der Krim" zugänglich zu machen'; eine riesige Breitspurbahn, die auf vier Meter breiten Gleisen als "fahrendes Hotel" Oberschlesien mit dem Donezbecken verbinden sollte; und, als Basis für die ganze Utopie dienend, die Versklavung der ansässigen Bevölkerung, insofern sie nicht rassisch "gesäubert " und deportiert wurde, um Platz für deutsche Siedler zu schaffen. All dies gehörte zu seiner Vision von Deutschlands "Platz an der Sonne“. Zur Utopie gehörte schließlich auch die Verewigung der Herrenrasse in Speers Monumentalbauten in Berlin - der künftigen „Germania“ - und München.

Wenn im Jahre 1926, als der zweite Band von „Mein Kampf“ veröffentlicht wurde, jemand prophezeit hätte, innerhalb von 15 Jahren stünde Deutschland in einem völkermörderischen Krieg gegen Rußland, in Polen würden Gaskammern errichtet, um die Juden in ganz Europa auszurotten, und außerdem würden Pläne entwickelt, Abermillionen von Slawen über die folgenden 25 Jahre nach Sibirien zu deportieren, um den Ostraum zu germanisieren, hätte man ihn wohl für wahnsinnig gehalten. Wenn er darüber hinaus 1926 behauptet hätte, daß derjenige, der dies zustandebringen würde, Adolf Hitler wäre - anscheinend kaum mehr als ein wilder Fanatiker, Extremist, Radikaler, politischer Außenseiter, demagogischer Hitzkopf - hätte man das Urteil wahrscheinlich nicht revidiert. Dennoch geschah dies alles nach 1933. Die Rassenutopie Hitlers, die man 1926 in einem modernen, hochentwickelten Staat und in einer Gesellschaft auf hohem kulturellen Niveau für wahnwitzig erklärt hätte, wurde $1941 / 42$ beinahe realisiert. Wie war das möglich? Die Rolle des sonderbaren Individuums Adolf Hitler gehört selbstverständlich ins Zentrum von jeglichem Erklärungsversuch. Aber ein plausibler Erklärungsversuch kann sich kaum auf Hitler beschränken, sondern muß über die Rolle seiner Person, so wichtig sie auch war, hinausgehen.

Ich kann hier nur in aller Kürze einige Punkte angeben, die, wie es mir scheint, Ansätze zu einer ausführlichen Erklärung anbieten. Ich nenne insgesamt zehn:

1. Ungewöhnlich tief und weitverbreitet war die umfassende Krise der deutschen Gesellschaft während der Zeit der Weimarer Republik. Politische Krisen sind in demokratischen, pluralistischen Systemen an sich keine Seltenheit. Wirtschaftliche Miseren geschehen sogar noch häufiger. Sie führen aber selten zum Zerfall des staatlichen Systems selbst und zur weitgehenden Verwandlung der Ge-

8 Adolf Hitler, Tischrede vom 17./18. September 1941, in: ders., Monologe im Hauptquartier 1941-1944. Die Aufzeichnungen Heinrich Heims, hrsg. von Werner Jochmann (Hamburg 1980) $62 \mathrm{f}$.

9 Ebd. 39 (vom 5./6. Juli 1941). 
sellschaft, nicht zuletzt, weil sie meistens Notlagen innerhalb des Systems sind, die sich aber nicht gegen das System als solches richten. Im Falle Deutschlands im Gegensatz z. B. zu Großbritannien oder den USA - herrschte eine Gesamtkrise der Gesellschaft und des Staates - eine Misere, die so umfassend war, daß sie auch die kulturellen Werte des Landes in ihrem Kern betraf. Diese Krise spitzte sich zwar weltweit in der Wirtschaftsdepression zu, hatte aber speziell in Deutschland langfristige Wurzeln, die in die Vorkriegszeit zurückreichten. Dies führte dazu, daß sie eigentlich durch die ganze Weimarer Zeit hindurch schwelte. Ein tief verankerter Kulturpessimismus, der wohl viel tiefgreifender und auch bedeutender war als in anderen Ländern wie Frankreich oder Italien, wo er ebenfalls zur Entstehung von faschistischen Bewegungen führte, war ein wichtiger Bestandteil, vor allem in intellektuellen Kreisen. Oswald Spenglers „Untergang des Abendlandes“ sprach eine solche Mentalität direkt an. Das weitverbreitete Gefühl der nationalen Erniedrigung, ein Gefühl, das Hitler selbst traumatisiert hatte, verstärkte dann, schon lange bevor der Nationalsozialismus politisch bedeutend wurde, die Bereitschaft in gebildeten Kreisen, so fern sie dem nationalistischen Pöbel auch standen, cine nationale Erneuerung - das Ende der „Dekadenz“ - herbeizuwünschen. Das war zwar kein Nationalsozialismus an sich; aber es schuf den Nährboden für die spätere Akzeptanz eines charismatischen Führers, der die Errettung der Nation verhieß. Die restlose Vernichtung der Gegner der nationalen Erneuerung gehörte zu dieser Botschaft und wurde auch von der immer größer werdenden Anhängerschaft des Nationalsozialismus weitgehend in Kauf genommen ${ }^{10}$.

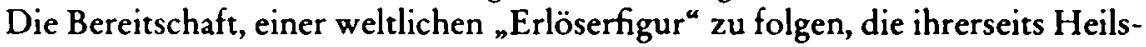
erwartungen wecken konnte, hatte nicht zuletzt mit der Glaubenskrise einer sich rasch modernisierenden Gesellschaft zu tun. Strukturell war diese Krise in vieler Hinsicht von denjenigen im übrigen Europa zu unterscheiden - und sie trug wesentlich zur Ausprägung einer spezifisch deutschen Form der Führererwartung bei.

2. Hitler selbst war natürlich absolut unerläßlich zur Realisierung der Rassenutopie - d. h. zur Verwirklichung einer sich kontinuierlich radikalisierenden und schließlich umfassenden genozidalen Judenpolitik. In entscheidenden Phasen - wie z. B. bei der Entstehung der Nürnberger Gesetze 1935, der Entfesselung des Novemberpogroms 1938 oder der Genesis der Endlösung 1941 - waren Hitlers Eingriffe und seine Ermächtigung bestimmend. Andererseits blieb er über lange Strecken hindurch relativ inaktiv in der Rassenpolitik: dennoch radikalisierte sich diese zusehends. Andere Kräfte arbeiteten offensichtlich „dem Führer entgegen “11.

Die Realisierung der Utopie wurde selbstverständlich durch die Verabsolutierung von Hitlers Machtstellung innerhalb des Regimes ermöglicht. Die Grundlage für seine Macht im Staate basierte wiederum in erster Linie darauf, daß es Hit-

10 Für die Attraktivität der politischen Gewalt s. Bernd Weisbrod, Violence and Sacrifice: Imagining the Nation in Weimar Germany, in: Hans Mommsen (Hrsg.), The Third Reich between Vision and Reality (Oxford, New York 2001) 5-21.

11 Rede von Werner Willikens, Niedersächsisches Staatsarchiv, Best. 131 Nr. 303, fol. 131v; Zitat bei Ian Kershaw, Hitler. Bd. 1, 1889-1936 (Stuttgart 1998) 665. 
ler gelungen war, eine politische Massenbewegung total zu dominieren, die sich einer ungewöhnlichen Herrschaftsform - der charismatischen Herrschaft - unterordnete und die utopischen Fernziele des Führers in Aktionismus konvertierte. Ab 1925 vermochte es Hitler, die NSDAP in eine Führerpartei zu verwandeln und sich selbst allmählich zum Sprachrohr der gesamten völkischen Bewegung zu machen. Er galt, nach verschiedenen internen Kämpfen und Auseinandersetzungen, als die Verkörperung der NS-Weltanschauung, als das Fundament der ideologischen Orthodoxie und als die legitimierende Instanz für die Zielrichtung und den Aktionismus der Bewegung. Die Fixpunke seiner persönlichen Weltanschauung dienten somit auch als Orientierungshilfen für die Aktionsrichtungen zunächst der NS-Bewegung und später des NS-Regimes. Mit der Verabsolutierung seiner Machtstellung im Staate ab 1933 gewann seine persönliche ideologische Triebkraft und Motivation zunehmenden Einfluß auf das gesamte Staatswesen - und schuf deswegen auch das Potential für die Verwirklichung der NS-Rassenutopien.

3. Die Rassenutopien waren allerdings keineswegs Hitlers Erfindung. Eher entsprach er in seiner Radikalität, Rhetorik und visionären Kraft Vorstellungen, die in weiten Kreisen der völkischen Bewegung zu Beginn der 20er Jahre die Runde gemacht hatten. Es gelang Hitler dadurch - meist unterstützt durch seine Bierhallen-Rhetorik - eine fanatische Anhängerschar hinter sich zu versammeln, die von völkischen Antipathien und $\mathrm{Haßgefühlen} \mathrm{genauso} \mathrm{besessen} \mathrm{war} \mathrm{wie} \mathrm{Hitler} \mathrm{selbst.}$ Diese Anhänger bildeten den Kern einer weltanschaulich voll überzeugten $\mathrm{Ge}$ folgschaft, die sich zu Hitlers treuer Paladinengruppe entwickelte. Beispielsweise waren die meisten Gauleiter, die sich sehr früh zu Hitler bekannten, später seine Vizekönige in den Provinzen wurden und die weltanschaulichen Richtlinien des Nationalsozialismus in politische Aktion umsetzten, etwa gleichaltrig mit Hitler; wie er hatten sie das Aufblühen des Antisemitismus in der kritischen Phase zwischen etwa 1916 und 1923 miterlebt - freilich noch zum größten Teil als Anhänger irgendeiner völkischen Gruppe, bis sie dann den Weg zu Hitler fanden. Der Beitrag dieser „alten Garde“ der Partei als extremen Hitler-Loyalisten und überzeugten Trägern der NS-Weltanschauung zur Implementierung der Rassenutopien kann kaum überschätzt werden. Auf eigene Faust, ohne auf Befehle zu warten, versuchten diese Radikalen, die Rassenutopien zu verwirklichen, die Judenverfolgung wo immer möglich voranzutreiben, die rassisch gesäuberte Gesellschaft in Deutschland zu realisieren, oder die schlimmste Germanisierungspolitik in den okkupierten Gebieten des Ostens mit aller Kraft und so eilig wie möglich durchzusetzen. Die ideologisch fanatisierte Gefolgschaft reichte natürlich viel weiter als die Gauleiter und andere Führungsgruppen in der NS-Bewegung. Eine neue statistische Untersuchung von etwa 1500 "Tätern", die an der Ausrottung der Juden direkt mitwirkten, hat m.E. überzeugend nachgewiesen, daß diese Täter weder "gewöhnliche Männer" (im Sinne von Christopher Browning) ${ }^{12}$ noch „gewöhnli-

12 Christopher Browning, Ganz normale Männer. Das Reserve-Polizeibataillon 101 und die „Endlösung“ in Polen (Reinbek 1993). 
che Deutsche ${ }^{\mu}$ (wie Daniel Goldhagen meinte) ${ }^{13}$ waren, sondern durchaus eingefleischte Nazis - eingefleischt deswegen, weil sie einen langen "Sozialisierungsprozeß" durchgemacht hatten, in dem Gewaltanwendung gegen politische Gegner und rassische Minderheiten eine wichtige Rolle spielten ${ }^{14}$.

4. Von ausschiaggebender Bedeurung für die Realisierung der Rassenutopien war ab 1933 die Verzahnung der SS, der ideologischen Elitetruppe der Bewegung, und der Polizei, dem staatlichen Kontroll- und Unterdrückungsapparat. Es bildete sich daraus unter der Leitung von Heinrich Himmler und Reinhard Heydrich eine große, vielschichtige Organisation, die sich rasch zum wichtigsten Herrschaftsinstrument des NS-Regimes entwickelte - nicht zuletzt, weil sie sich als unmittelbares Ausführungsorgan des Führerwillens verstand und ihre Aufgaben rein ideologisch auffaßte. Wie Werner Best es 1936 ausdrückte, hatte nder nationalsozialistische Führerstaat zum ersten Mal in Deutschland eine ... Einrichtung (entwickelt), die den politischen Gesundheitszustand des deutschen Volkskörpers sorgfältig überwacht, jedes Krankheitssymptom rechtzeitig erkennt und die Zersiörungskeime ... feststellt und mit jedem geeigneten Mittel beseitigt "${ }^{15}$. Die Führung dieser auf ganz neuer, radikaler Basis konzipierten Polizei lag in den Händen von Männern wie Best, die, wie Ulrich Herbert und andere gezeigt haben, Anfang der 20er Jahre als Studenten in deutschen Universitäten das völkische Gedankengut voll aufgesogen und verinnerlicht hatten ${ }^{16}$. Später befanden sich diese hoihintelligenten, gebildeten und ideologisch motivierten Technokraten der Macht in der Lage, die propagandistisch dargelegten visionären Fernziele Hitlers in Planungsaufgaben und polizeiliche Maßnahmen umzusetzen. In den Wochen nach der sogenannten "Reichskristallnacht ", in einer entscheidenden Phase, in der die Hanthabung der Judenpolitik weitgehend der SS übergeben wurde, wurden die Weichen für eine Entwicklung gestellt, die nun konsequent zum Völkermord führte. Die Organisation, die imstande war, die Rassenutopie zu verwirklichen, war inzvischen geschaffen. Eine Mentalität, die den Völkermord als Mittel billigte, war innerhalb dieser Organisation bereits vorhanden gewesen. Das SS-Organ „Dai Schwarze Korps“ behauptete im November 1938, es bestünde die Notwendigkeit, "die jüdische Unterwelt genau so auszurotten, wie wir in unserem Ordnunysstaat Verbrecher eben auszurotten pflegen: mit Feuer und Schwert! Das

${ }^{13}$ Danie! J. Goldhagen, Hitlers willige Vollstrecker. Ganz gewöhnliche Deutsche und der Holocaux (Berlin 1996).

14 Michad Mann, Were the Perpetrators of Genocide "Ordinary Men" or "Real Nazis"? Results from Fifteen Hundred Biographies, in: Holocaust and Genocide Studies 14 (2000) 331-66.

15 Hans Iuchbeim u. a., Anatomie des SS-Staates, Bd. 2 (Olten, Freiburg i. Br.) $50 \mathrm{f}$.

$16 \mathrm{Vgl}$. Urich Herbert, "Generation der Sachlichkeit". Die völkische Studentenbewegung der frühen zwanziger Jahre in Deutschland, in: Frank Bajabor, Werner Johe, Uwe Lobalm (Hrsg.), Zivilisation und Barbarei (Hamburg, 1991) 115-44; ders., Best. Biographische Studien üer Radikalismus, Weltanschauung und Vernunft 1903-1989 (Bonn 1996); jetzt, vor allem, Mchael Wildt, Generation des Unbedingten. Das Führungskorps des Reichssicherheitshaupamtes (Hamburg 2002). 
Ergebnis wäre das tatsächliche und endgültige Ende des Judentums in Deutschland, seine restlose Vernichtung. " 17

5. Die Verschmelzung von SS und Polizei deutet auf einen weiteren, wichtigen Prozeß hin, der die Realisierung der Rassenutopie ermöglichte: den Prozeß, daß sämtliche Machtinstrumente eines modernen Staates sich der nationalen Erlösungsideologie, die in Hitler verkörpert war, zunehmend unterordneten. Das ideologische Fernziel der "Entfernung der Juden“ infiltrierte ab 1933 alle staatlichen Instanzen und durchdrang jede Facette des öffentlichen Lebens im Dritten Reich. Ohne das aktive Mitwirken der staatlichen Bürokratie an der Ausgrenzung und Verfolgung der Juden - und anderer diskriminierter Gruppen - wäre die NSRassenutopie völlig unrealisierbar geblieben. Der Staatsapparat verwandelte die losen Propaganda- und Kampfparolen Hitlers und anderer NS-Führer in eine Unmenge sich ständig radikalisierender Gesetze, Verordnungen und Erlasse. Die verschiedenen Behörden überboten sich geradezu in dem Versuch, die diskriminierende Gesetzgebung in ihren Domänen möglichst effizient durchzusetzen. Die Teilnahme der Staatssekretäre an der Wannsee-Konferenz war ein vielsagendes Indiz dafür, daß die Machtinstrumente eines modernen Staates in die Verwirklichung der mörderischen Utopien der NS-Führung nun vollends verwickelt waren. In welchem Ausmaß die "legal-rationale" Autorität des modernen Staates - um die Terminologie Max Webers zu verwenden - bereit war, sich den in der "charismatischen Autorität " Hitlers verkörperten utopischen Fernzielen unterzuordnen, zeigt sich in dem Kommentar des bedeutenden Verfassungstheoretikers Ernst Rudolf Huber, der gegen Ende der 30er Jahre bezeichnenderweise schrieb: Es sei nnicht möglich, die Gesetze des Führers an einer ihnen übergeordneten Rechtsidee zu messen, da jedes Führergesetz unmittelbar Ausdruck dieser völkischen Rechtsidee ist ${ }^{\prime 18}$. Das Rechtssystem sowie die staatliche Bürokratie wurden somit zu nichts als Werkzeugen des Führerwillens und damit zu Mitteln zur Realisierung der NS-Rassenutopien.

6. Die NS-Rassenutopien waren zwar der zentrale Bestandteil, aber nicht das einzige Element eines weitgreifenden utopischen Projekts des nationalen Aufbaus, das sehr große Anziehungskraft auf breite Kreise der Bevölkerung, einschließlich der nicht-nationalsozialistischen Eliten, ausübte. Wie schon erwähnt, war der Nährboden für die breite Akzeptanz dieses propagandistisch ständig ausgeschlachteten utopischen Vorhabens bereits vorhanden, bevor die Krise der Weimarer Demokratie der nationalen Erlösung, wie sie der charismatische Führer Hitler versprach, mehr Attraktivität verlieh. Unter Jugendlichen insbesondere konnte Hitler vorhandene utopische Zukunftsvorstellungen ansprechen und ausnutzen. Die anfänglichen erstaunlichen "Erfolge“ Hitlers hatten dann nach 1933 zur Folge, daß sich gleichermaßen die Massen - oder zumindest, trotz vielerlei Dissens und Besorgnissen, die große Mehrheit der Bevölkerung - wie

17 Vgl. „Das Schwarze Korps“ (24. November 1938).

18 Emst Rudolf Huber, Verfassungsrecht des Großdeutschen Reiches (Hamburg 21939) 230. 
auch die Funktionseliten der Wehrmacht, der Wirtschaft, der staatlichen Bürokratie und darüber hinaus die intellektuellen Eliten - die späteren "Vordenker der Vernichtung ${ }^{19}$-, immer enger an Hitler banden. Bleiben wir zunächst bei den Eliten.

Hitlers weltanschauliche Fixpunkte - „Entfernung der Juden“, Erlangung von Lebensraum, Gewinnung des Weltmachtstatus' - standen in keinerlei Widerspruch zu den handfesten Interessen der Funktionseliten. Im Gegenteil: Als Fernziele waren sie so vage angelegt, daß sie mehr oder weniger zu allen Interessen paßten. Die „unangenehmen“ Erscheinungen des Regimes - seine Schattenseite konnten außerdem als notwendiger Preis für den gelungenen Aufbau der Nation in Kauf genommen werden. Der symbolhafte Charakter des Lebensraummottos Martin Broszat sprach von einer ideologischen „Metapher ${ }^{40}$ - zusammen mit der Vision der deutschen Vorherrschaft in Europa, später in der ganzen Welt, vereinigte die unterschiedlichen Interessen der Eliten. Hitler öffnete neue Möglichkeiten der Phantasie und setzte so Kräfte frei. Ingenieure oder Ärzte z. B. konnten ihre eigenen Utopien auf einmal für realisierbar halten, ohne sich von „lästigen“ finanziellen Einschränkungen oder den Menschenrechtskonventionen demokratischer Systeme gezügelt zu fühlen.

Insbesondere die Streitkräfte und die Wirtschaft sahen ihre eigenen Interessen durch die Aufbau- und Expansionspolitik des Nationalsozialismus vertreten und gefördert. Die „Entfernung der Juden“ störte diese Gruppen nicht und konnte sogar teilweise, wie bei der "Arisierung “, große Gewinne bringen. Insofern wurde bis in den Krieg hinein die Judenverfolgung, wenn sie nicht sogar zu sektoralen Interessen paßte, vielfach als Nebenerscheinung angesehen. Auch in Polen störte die schreckliche Eskalation der Judenverfolgung kaum. Die Einwände von einigen Generälen gegen die Barbarei der SS blieben hier reine Ausnahmen. Und bis zu diesem Zeitpunkt hatte die Judenpolitik bereits eine weitgehende Eigendynamik entwickelt, die dann, solange das NS-Regime existierte, nicht mehr gestoppt werden konnte, selbst wenn sie sich schließlich direkt gegen ökonomische Grundgesetze richtete. Zur Entfaltung dieser Eigendynamik hatten die Funktionseliten selbst ihren unentbehrlichen Beitrag geleistet - zumindest, bis das Stadium des grenzenlosen Völkermordes begann.

7. Daniel Goldhagen nannte die Deutschen Hitlers "willige Vollstrecker". Deutschland war nach seiner Interpretation einmalig, weil ein „eliminatorischer Antisemitismus" in der politischen Kultur seit dem 19. Jahrhundert als vorherrschendes Leitmotiv fungiert habe, so daß die Vernichtungspolitik Hitlers eigentlich nur den Wünschen der Bevölkerung insgesamt entsprochen habe ${ }^{21}$. Die Inter-

19 Götz Aly, Susanne Heim, Vordenker der Vernichtung. Auschwitz und die deutschen Pläne für eine neue europäische Ordnung (Frankfur a.M. 1991).

20 Martin Broszat, Soziale Motivation und Führer-Bindung des Nationalsozialismus, in: VfZ 18 (1970) 392-409, hier: 407; im folgenden zitiert; Broszat, Soziale Motivation.

21 S. o., Anm. 12. 
pretation ist m.E. zu Recht als grobe Simplifizierung heftig kritisiert worden ${ }^{22}$. Die Einzigartigkeit Deutschlands bestand nicht in einer breit akzeptierten, jahrzehntenlang anhaltenden Sehnsucht nach der "Eliminierung" der Juden, sondern darin, daß ab 1933 eine potentiell genozidale Elite die Macht im Staat übernehmen konnte und nun in der Lage war, die in völkischen Kreisen vorhandene utopische Vorstellung von der "Entfernung der Juden" zum Kernbestand der staatlichen Ideologie und Politik zu machen. Erst jetzt breitete sich ein regelrechter „eliminatorischer “ Antisemitismus aus. Unter einer rücksichtslosen, fanatisierten Staatsführung, die jede nennenswerte oppositionelle Handlung brutal niederschlägt, ist es ein ganz normales Verhalten, passiv zu bleiben, die eigene Freiheit, vielleicht sogar den Kopf, nicht riskieren zu wollen. So erklärt sich zum Teil - aber nur zum Teil - auch die mangelnde Bereitschaft in Deutschland, gegen die Judenverfolgung Stellung zu nehmen. Passivität und moralische Indifferenz, meist ohnehin mit antisemitischen Vorurteilen verbunden, konnten aber dem radikalen Judenhaß einer Minderheit, die noch dazu staatliche Unterstützung im Rücken hatte, keinen Einhalt bieten. Noch schlimmer: Wenn nicht alles trügt, war die Unterdrückung von "Gegnern" sowie die Ausgrenzung von Juden und anderen "unerwünschten" Minderheiten bei der großen Masse der Bevölkerung durchaus populär ${ }^{23}$. Nicht nur dies: Unzählige Deutsche wirkten freiwillig bei der Verfolgung mit. Die NSPolitik bot die Chance, auf Kosten der Juden zu profitieren - also z. B. eine neue Wohnung zu bekommen, einen Geschäftskonkurrenten auszuschalten usw. Auf vielerlei Art und Weise zeigt sich, daß viele Leute versuchten, direkt oder indirekt "im Sinne des Führers" zu arbeiten, indem sie unaufgefordert dem bekannten Ziel Hitlers, die Juden zu „entfernen“, entgegenkamen; damit lieferten sie ihren eigenen Beitrag zur Spirale der Diskriminierung. Und mit der eskalierenden Verfolgung rückte die Erfüllung von der utopischen Vision eines Deutschlands, dann eines Europas, ohne Juden allmählich in greifbare Nähe.

8. Von kaum überschätzbarer Bedeutung für die "Realisierung des Utopischen“ (Hans Mommsen) ${ }^{24}$ war, daß die NS-Propaganda rassische Feindbilder und das Image von wirklichen oder vermeinten militärischen Feinden Deutschlands miteinander verschmolz. Für die Juden war dies doppelt fatal, denn sie wurden in der NS-Lehre und -Propaganda sowohl mit dem Bolschewismus, also dem Erzfeind in Moskau, als der größten Gefahr für die weitere Existenz des Abendlandes gleichgesetzt, als auch mit dem Kapitalismus, dem mächtigen Feind in der City of London und in Wall Street in New York identifiziert. Hitlers berüchtigte "Prophezeiung" vom 30. Januar 1939, daß im Fall eines Kriegs die Juden in Europa vernichtet würden, betonte (nach dieser grotesken Sicht der Dinge) die Verant-

22 Siehe z. B. Julius $H$. Schoeps, Ein Volk von Mördern? Die Dokumentation zur GoldhagenKontroverse um die Rolle der Deutschen im Holocaust (Hamburg 1996).

${ }^{23}$ Siehe Robert Gellately, Backing Hitler. Consent and Coercion in Nazi Germany (Oxford 2001).

24 S. o., Anm. 4. 
wortung der Juden für die Entfachung eines neuen Weltbrandes ${ }^{25}$. Die Juden wurden daher nicht nur als die größte innenpolitische Kraft der Zersetzung, sondern als internationale Todfeinde in einem Kampf um Dasein oder Untergang der Nation angesehen. Für die Explosion des Völkermords in der Sowjetunion ab dem 22. Juni 1941 war ihre Identifizierung mit dem Bolschewismus - nicht zuletzt innerhalb der Wehrmacht - von höchster Bedeutung.

9. Es war daher kein Zufall, daß die Ermordung der Juden - die Realisierung der NS-Rassenutopie - im Rahmen eines Krieges, der einem ideologischen Kreuzzug gleichkam, stattfand. Hitler hatte diesen Krieg seit den $20 \mathrm{er}$ Jahren gewollt. $\mathrm{Da}$ nach seiner perversen Weltanschauung der Endsieg nur mit der totalen Vernichtung derjenigen, die den Krieg verursacht hatten, also der Juden, errungen werden konnte, war ihre Ermordung im Krieg nur folgerichtig. Die „Endlösung der Judenfrage“, wie sie ja tatsächlich umgesetzt wurde, entwickelte sich erst nach viel Improvisation und in verschiedenen Eskalationsschüben zwischen dem Überfall auf die UdSSR und dem Frühjahr 1942. Hitlers direkte Rolle bleibt auch hier meist im Dunkeln, nicht zuletzt weil er dafür sorgte, daß auch in seiner engsten Umgebung im Führerhauptquartier der Judenmord tabuisiert wurde. Außer Zweifel steht aber, daß er seine Umgebung zu den wichtigsten Schritten persönlich ermächtigte; darüber hinaus ist auch seine Kenntnis und Billigung des Geschehens unstrittig. Und nach acht Jahren, in denen die Juden weit und breit verteufelt und zu Unmenschen deklariert worden waren, in denen eine genozidale Mentalität in den wichtigsten Herrschaftsinstrumenten gezüchtet worden war, nimmt es nicht Wunder, daß es an "willigen Vollstreckern“ nicht mangelte.

10. Schließlich konnte die NS-Rassenutopie realisiert werden, weil diejenigen Gegner des Regimes, die in hohen Stellungen fungierten und somit das Potential und die Mittel besaßen, mit der Person Hitlers sowohl den Hauptexponenten der skizzierten Utopie als auch die Schlüsselfigur im genozidalen Prozeß überhaupt zu beseitigen, zu einem solchen Vorgehen weder willens noch in der Lage waren, his es schließlich zu spät war. Warum dies nicht geschah, ist natürlich ein anderes und äußerst kompliziertes Thema, das seiner eigenen ausführlichen Erklärung bedarf. Hier bleibt nur festzuhalten: Die fehlende Bereitschaft, Hitler zu stoppen, aus welchen Motivationen sie sich auch immer speiste, ermöglichte schließlich die Realisierung des Utopischen.

In allen Stadien der Realisierung von der NS-Rassenutopie war Hitler die „unerläßliche Integrationsfigur und Drehscheibe des Geschehens" (Broszat) ${ }^{26}$. Aber gerade die Tatsache, daß der lange Radikalisierungsprozeß, der schließlich in der "Endlösung" kulminierte, über lange Strecken hindurch ohne direkte Eingriffe Hitlers stattfand, daß Hitler sich „mit der konkreten Durchsetzung des antisemitischen Programms " kaum befaßte ${ }^{27}$, und daß es wohl weder einen schriftlichen

25 Das Zitat findet sich bei Hitler. Reden und Proklamationen 1932-1945 (Wiesbaden 1973) 1058.

26 Broszat, Soziale Motivation 409.

27 Mommsen, Realisierung des Utopischen 390. 
noch einen direkten mündlichen Befehl Hitlers zum Judenmord gegeben hat, $\mathbf{z w i n g t}$ den heutigen Betrachter dazu, nicht nur die Person Hitlers, sondern auch die funktionale Bedeutung seiner charismatischen Herrschaft in den Vordergrund zu stellen. Jede Analyse, die erklären will, wie die NS-Rassenutopie verwirklicht werden konnte, muß deswegen insbesondere zwei Phänomene berücksichtigen: Erstens, daß eine äußerst ungewöhnliche Herrschaftsform - die charismatische Herrschaft - die für den modernen Staat typische legal-bürokratischen Herrschaftsform überlagerte, und zweitens, daß die Machtinstrumente des Staates sich zu Werkzeugen einer nationalen Erlösungsideologie reduzieren ließen.

Die charismatische Herrschaft Hitlers, die wie bereits erwähnt unverkennbar pseudoreligiöse Züge aufwies und durch ihre utopische Zukunftsvision gefährliche Heilserwartungen in der Bevölkerung einer modernen Gesellschaft weckte, war in vielem atavistisch. Sie beruhte nichtsdestoweniger zuletzt auf einem wichtigen Merkmal der Moderne, nämlich auf der wohl erst seit dem Ende des 18. Jahrhunderts existierenden Überzeugung, daß nicht Gott, sondern der Mensch selbst imstande sei, die Zukunft der Menschheit zu gestalten und deswegen auch das menschliche Glück nicht erst im Himmelreich zu finden sei. In der Moderne schien der Himmel auf Erden denkbar und allmählich aufgrund der Entwicklung von Naturwissenschaften und Technologie auch realisierbar. Der nächste Denkschritt, daß diejenigen, die diese Realisierung gefährdeten, die nicht zur neuen Utopie zählten, nverschwinden “ und damit letztlich physisch ausgerottet werden müßten, war logisch; er brauchte aber, um vorstellbar $\mathrm{zu}$ werden, das Menschenschlachten des Ersten Weltkrieges, das Vorhandensein von neuen Ideologien der Vernichtung und die breite Akzeptanz von massiver staatlicher Gewalt gegen Mitmenschen. Insofern stellte die Menschenvernichtung des Nationalsozialismus durchaus einen Teil der Moderne dar, einen Teil von modernen und utopischen Zukunftsvorstellungen, die sich aus den erläuterten Gründen im Deutschland der NS-Zeit katastrophal entwickeln konnten. 